\title{
GENERATORS OF THE EISENSTEIN-PICARD MODULAR GROUP
}

\author{
JIEYAN WANG, YINGQING XIAO ${ }^{凶}$ and BAOHUA XIE
}

(Received 11 November 2010; accepted 7 October 2011)

Communicated by M. G. Cowling

\begin{abstract}
We prove that the Eisenstein-Picard modular group $\mathrm{SU}\left(2,1 ; \mathbb{Z}\left[\omega_{3}\right]\right)$ can be generated by four given transformations.
\end{abstract}

2010 Mathematics subject classification: primary 32M05; secondary 22E40, 32M15.

Keywords and phrases: complex hyperbolic space, Picard modular group.

\section{Introduction}

The Picard modular groups $\mathrm{SU}\left(2,1 ; O_{d}\right)$ are the subgroups of $\mathrm{SU}(2,1)$ with entries in $O_{d}$. Here $O_{d}$ is the ring of algebraic integers in the imaginary quadratic number field $\mathbb{Q}(i \sqrt{d})$ for any positive square-free integer $d$. If $d \equiv 1,2 \bmod 4$, then $O_{d}=\mathbb{Z}[i \sqrt{d}]$, and if $d \equiv 3 \bmod 4$, then $O_{d}=\mathbb{Z}\left[\frac{1}{2}(1+i \sqrt{d})\right]$. It is well known that the ring $O_{d}$ is Euclidean for positive square-free integers $d$ if and only if $d=1,2,3,7,11$.

The Picard modular groups $\mathrm{SU}\left(2,1 ; O_{d}\right)$ are the simplest arithmetic lattices in $\mathrm{SU}(2,1)$. In the case that $d \equiv 3 \bmod 4$, the ring $O_{d}$ can be described as $O_{d}=$ $\mathbb{Z}\left[\frac{1}{2}(-1+i \sqrt{d})\right]$. Here the ring $\mathbb{Z}\left[\frac{1}{2}(-1+i \sqrt{d})\right]$ is isomorphic to the ring $\mathbb{Z}\left[\frac{1}{2}(1+i \sqrt{d})\right]$. The Picard modular groups can also be denoted by $\mathrm{SU}\left(2,1 ; \mathbb{Z}\left[\omega_{d}\right]\right)$ if we let $\omega_{d}=$ $\frac{1}{2}(-1+i \sqrt{d})$.

In general the presentation of a group can be obtained by constructing an explicit fundamental domain. Falbel and Parker (see [4]) studied the Eisenstein-Picard group $\mathrm{SU}\left(2,1 ; \mathbb{Z}\left[\omega_{3}\right]\right)$ and gave a system of generators and the corresponding presentation for this lattice. They similarly obtained a presentation of the Gauss-Picard modular group $\mathrm{SU}(2,1 ; \mathbb{Z}[i])$ in [3].

In [2] the authors used a constructive method to obtain a finite system of generators for the Gauss-Picard modular group $\mathrm{SU}(2,1 ; \mathbb{Z}[i])$. More precisely, they

This work was partially supported by The National Natural Science Foundation of China (No. 11071059). Xie was also supported by Hunan University (No. 531107040021).

(c) 2012 Australian Mathematical Publishing Association Inc. 1446-7887/2012 \$16.00 
proved that the Gauss-Picard modular group $\mathrm{SU}(2,1 ; \mathbb{Z}[i])$ can be generated by four transformations: two Heisenberg translations, a rotation and an involution. Their description was applied to instanton corrections in string theory in [1].

It would be interesting to know whether the method used in [2] can be extended to the Euclidean Picard modular groups $\mathrm{SU}\left(2,1 ; O_{d}\right)$ for $d=2,3,7,11$. In this note we show that the method used in [2] can applied to the Eisenstein-Picard modular group $\mathrm{SU}\left(2,1 ; \mathbb{Z}\left[\omega_{3}\right]\right)$ and obtain a simple description of this group in terms of its generators. Recently, using a different method, Zhao found generators of the Euclidean Picard modular groups $\mathrm{SU}\left(2,1 ; O_{d}\right)$ for $d=2,7,11$ in [11].

In this paper we find a connection between the generators of the Eisenstein-Picard modular group $\mathrm{SU}\left(2,1 ; \mathbb{Z}\left[\omega_{3}\right]\right)$ given in [4] and the generators given in this note. This connection leads to a new presentation of the lattice.

This paper is organized as follows. In Section 2 we introduce some basic general definitions and results from complex hyperbolic geometry and the Picard modular groups. The main result and its proof appear in Section 3.

\section{Preliminaries}

In this section we recall some basic definitions and results from complex hyperbolic geometry which can be found, for example, in [2, 7-10].

Let $\mathbb{C}^{2,1}$ denote the three-dimensional complex vector space $\mathbb{C}^{3}$ equipped with the Hermitian form

$$
\langle z, w\rangle=w^{*} J z=z_{1} \bar{w}_{3}+z_{2} \bar{w}_{2}+z_{3} \bar{w}_{1}
$$

of signature $(2,1)$. Here the matrix $J$ is defined by

$$
J=\left(\begin{array}{lll}
0 & 0 & 1 \\
0 & 1 & 0 \\
1 & 0 & 0
\end{array}\right)
$$

and the vectors $z$ and $w$ have the form

$$
z=\left(z_{1}, z_{2}, z_{3}\right)^{t}, \quad w=\left(w_{1}, w_{2}, w_{3}\right)^{t}
$$

where we denote by $x^{t}$ the transpose of the vector $x$.

Let $z \in \mathbb{C}^{2,1}$. Then $\langle z, z\rangle$ is real. Thus we can define subsets $V_{0}, V_{-}$of $\mathbb{C}^{2,1}$ by

$$
\begin{aligned}
& V_{0}=\left\{z \in \mathbb{C}^{2,1}-\{0\} \mid\langle z, z\rangle=0\right\}, \\
& V_{-}=\left\{z \in \mathbb{C}^{2,1} \mid\langle z, z\rangle\langle 0\} .\right.
\end{aligned}
$$

The complex hyperbolic space $\mathbf{H}_{\mathbb{C}}^{2}$ is defined to be the complex projective subspace $\mathbb{P}\left(V_{-}\right)$equipped with the Bergman metric where

$$
\mathbb{P}: \mathbb{C}^{2,1}-\{0\} \rightarrow \mathbb{C P}^{2}
$$

is the canonical projection onto the complex projective space. The boundary of the complex hyperbolic space is defined to be $\partial \mathbf{H}_{\mathbb{C}}^{2}=\mathbb{P}\left(V_{0}\right)$. 
Using nonhomogeneous coordinates, we see that the complex hyperbolic space $\mathbf{H}_{\mathbb{C}}^{2}$ is equal to the Siegel domain

$$
\left\{\left.\left[\begin{array}{c}
z_{1} \\
z_{2} \\
1
\end{array}\right] \in \mathbb{C P}^{2}\left|2 \operatorname{Re}\left(z_{1}\right)+\right| z_{2}\right|^{2}<0\right\} .
$$

Let $\mathfrak{N}$ denote the Heisenberg group which is equal to the set $\mathbb{C} \times \mathbb{R}$ with the product

$$
\left(z_{1}, t_{1}\right)\left(z_{2}, t_{2}\right)=\left(z_{1}+z_{2}, t_{1}+t_{2}+2 \operatorname{Im}\left(z_{1} \bar{z}_{2}\right)\right) .
$$

Then $\mathbf{H}_{\mathbb{C}}^{2}$ can be parameterized in horospherical coordinates by $(z, t, u) \in \mathfrak{N} \times \mathbb{R}^{+}$with the connection map

$$
(z, t, u) \rightarrow\left[\begin{array}{c}
\left(-|z|^{2}-u+i t\right) / 2 \\
z \\
1
\end{array}\right]
$$

The boundary of the complex hyperbolic space $\partial \mathbf{H}_{\mathbb{C}}^{2}$ can be identified with the onepoint compactification $\overline{\mathfrak{N}}=\mathfrak{N} \cup\left\{q_{\infty}\right\}$ by the stereographic projection. Here $q_{\infty}=$ $(1,0,0)^{t}$ denotes the point at infinity.

The holomorphic isometry group of $\mathbf{H}_{\mathbb{C}}^{2}$ is $\mathrm{PU}(2,1)$. Recall that $\mathrm{PU}(2,1)$ is the projectivization of the special unitary group $\mathrm{SU}(2,1)$ that preserves the above Hermitian form. The matrix $G=\left(g_{j k}\right)_{j, k=1}^{3} \in \mathrm{SU}(2,1)$ satisfies the condition

$$
G^{*} J G=J .
$$

Here $G^{*}$ denotes the conjugate transpose of the matrix $G$ and the determinant of the matrix $G$ is normalized to be equal to 1 . The Picard modular groups $\operatorname{SU}\left(2,1 ; O_{d}\right)$ are discrete holomorphic automorphism subgroups of $\mathbf{H}_{\mathbb{C}}^{2}$. The stabilizer subgroup $\Gamma_{\infty}$ of $q_{\infty}$ in $\mathrm{SU}(2,1)$ contains three important classes of elements, namely the Heisenberg translations, dilations and rotations.

The Heisenberg translation by $(z, t) \in \partial \mathbf{H}_{\mathbb{C}}^{2}$ is given by the matrix

$$
N_{(z, t)} \equiv\left(\begin{array}{ccc}
1 & -\bar{z} & \left(-|z|^{2}+i t\right) / 2 \\
0 & 1 & z \\
0 & 0 & 1
\end{array}\right) .
$$

The two Heisenberg translations $N_{\left(z_{1}, t_{1}\right)}$ and $N_{\left(z_{2}, t_{2}\right)}$ have product

$$
N_{\left(z_{1}, t_{1}\right)} \circ N_{\left(z_{2}, t_{2}\right)}=N_{\left(z_{1}+z_{2}, t_{1}+t_{2}+2 \operatorname{Im}\left(z_{1} \bar{z}_{2}\right)\right)}
$$

which is the Heisenberg translation corresponding to the product of the two points $\left(z_{1}, t_{1}\right)$ and $\left(z_{2}, t_{2}\right)$ in the Heisenberg group $\mathfrak{N}$.

The Heisenberg rotation by $\beta \in \mathbb{S}^{1}$ is given by the matrix

$$
M_{\beta} \equiv\left(\begin{array}{ccc}
1 & 0 & 0 \\
0 & \beta & 0 \\
0 & 0 & 1
\end{array}\right)
$$


The Heisenberg dilation by $\lambda \in \mathbb{R}^{+}$is given by the matrix

$$
A_{\lambda} \equiv\left(\begin{array}{ccc}
\lambda & 0 & 0 \\
0 & 1 & 0 \\
0 & 0 & \lambda^{-1}
\end{array}\right)
$$

The holomorphic involution $R$ swaps the point $q_{0}=(0,0) \in \partial \mathbf{H}_{\mathbb{C}}^{2}$ and the point at infinity $q_{\infty}$. It is given by the matrix

$$
R \equiv\left(\begin{array}{rrr}
0 & 0 & 1 \\
0 & -1 & 0 \\
1 & 0 & 0
\end{array}\right)
$$

Using the Langlands decomposition, any element $P \in \Gamma_{\infty}$ can be decomposed into a product of a Heisenberg translation, a dilation and a rotation. Thus all elements of $\Gamma_{\infty}$ can be written in the form

$$
P=\left(\begin{array}{ccc}
p_{11} & p_{12} & p_{13} \\
0 & p_{22} & p_{23} \\
0 & 0 & p_{33}
\end{array}\right)=N_{(z, t)} A_{\lambda} M_{\beta}=\left(\begin{array}{ccc}
\lambda & -\beta \bar{z} & \frac{1}{2}\left(-|z|^{2}+i t\right) \lambda \\
0 & \beta & \lambda^{-1} z \\
0 & 0 & \lambda^{-1}
\end{array}\right)
$$

The parameters satisfy the corresponding conditions.

Equation (2.1) tells us that all elements of $\Gamma_{\infty}$ are upper triangular. However, the following lemma gives a more precise characterization of the elements of $\Gamma_{\infty}$. We omit the proof since it is similar to that of [2, Lemma 1].

Lemma 2.1. Let $G=\left(g_{j k}\right)_{j, k=1}^{3} \in \mathrm{SU}(2,1)$. Then $G \in \Gamma_{\infty}$ if and only if $g_{31}=0$.

In [5, 6] it is shown that the Langlands decomposition (2.1) can also be used to parameterize a holomorphic automorphism $G=\left(g_{j k}\right)_{j, k=1}^{3}$ which is not in the subgroup $\Gamma_{\infty}$. Let $N_{G\left(q_{\infty}\right)}$ denote the Heisenberg translation which maps $q_{0}$ to $G\left(q_{\infty}\right)$. Then the transformation $P \equiv R N_{G(\infty)}^{-1} G$ belongs to $\Gamma_{\infty}$. Hence there are a Heisenberg translation $N$, a dilation $A$ and a rotation $M$ satisfying the equation

$$
G=N_{G(\infty)} R P=N_{G(\infty)} R N A M
$$

The transformations $N$ and $P$ in the decomposition of $G$ are not necessarily in the Picard modular groups $\mathrm{SU}\left(2,1 ; O_{d}\right)$ even if $G \in \mathrm{SU}\left(2,1 ; O_{d}\right)$. It is clear that the entries of $N$ and $P$ are not necessarily integers in the ring $O_{d}$.

\section{Main result and proof}

We use the notation $\operatorname{SU}\left(2,1 ; \mathbb{Z}\left[\omega_{3}\right]\right)$ to denote the Eisenstein-Picard modular group with $\omega_{3}=(-1+i \sqrt{3}) / 2$. In this section we extend the techniques of [2] to prove the following theorem. 
Theorem 3.1. The Picard modular group $\mathrm{SU}\left(2,1 ; \mathbb{Z}\left[\omega_{3}\right]\right)$ is generated by the Heisenberg translations

$$
N_{\left(\omega_{3}, \sqrt{3}\right)}=\left(\begin{array}{ccc}
1 & -\bar{\omega}_{3} & \omega_{3} \\
0 & 1 & \omega_{3} \\
0 & 0 & 1
\end{array}\right), \quad N_{(1, \sqrt{3})}=\left(\begin{array}{ccc}
1 & -1 & \omega_{3} \\
0 & 1 & 1 \\
0 & 0 & 1
\end{array}\right),
$$

the rotation

$$
M_{-\omega_{3}}=\left(\begin{array}{ccc}
1 & 0 & 0 \\
0 & -\omega_{3} & 0 \\
0 & 0 & 1
\end{array}\right)
$$

and the involution

$$
R=\left(\begin{array}{rrr}
0 & 0 & 1 \\
0 & -1 & 0 \\
1 & 0 & 0
\end{array}\right)
$$

In order to prove this theorem we first characterize the elements of the stabilizer subgroup $\Gamma_{\infty}$ of $q_{\infty}$ in the Picard modular group $\mathrm{SU}\left(2,1 ; \mathbb{Z}\left[\omega_{3}\right]\right)$.

Lemma 3.2. Let $\Gamma_{\infty}\left(2,1 ; \mathbb{Z}\left[\omega_{3}\right]\right)$ be the stabilizer subgroup of $q_{\infty}$ in $\mathrm{SU}\left(2,1 ; \mathbb{Z}\left[\omega_{3}\right]\right)$. Then any element $P \in \mathrm{SU}\left(2,1 ; \mathbb{Z}\left[\omega_{3}\right]\right)$ lies in $\Gamma_{\infty}\left(2,1 ; \mathbb{Z}\left[\omega_{3}\right]\right)$ if and only if the parameters in the Langlands decomposition of $P$ satisfy the conditions

$$
\lambda=1, \quad t \in \sqrt{3} \mathbb{Z}, z \in \mathbb{Z}\left[\omega_{3}\right], \beta= \pm 1, \pm \omega_{3}, \pm \omega_{3}^{2}
$$

and the integers $t / \sqrt{3}$ and $|z|^{2}$ have the same parity.

Proof. It is quite easy to see that $\lambda=1$. Considering the Langlands decomposition when $P \in \Gamma_{\infty}\left(2,1 ; \mathbb{Z}\left[\omega_{3}\right]\right)$ allows us to deduce that $|\beta|=1, z \in \mathbb{Z}\left[\omega_{3}\right]$ and $t \in \sqrt{3} \mathbb{Z}$. Since $\frac{1}{2}\left(-|z|^{2}+i t\right) \in \mathbb{Z}\left[\omega_{3}\right], t / \sqrt{3} \in \mathbb{Z}$ and $|z|^{2} \in \mathbb{Z}$, the integers $t / \sqrt{3}$ and $|z|^{2}$ have the same parity. As $\omega_{3}$ is a cube root of unity it follows that $\beta= \pm 1, \pm \omega_{3}$ or $\pm \omega_{3}^{2}$.

Proposition 3.3. Let $\Gamma_{\infty}\left(2,1 ; \mathbb{Z}\left[\omega_{3}\right]\right)$ be the stabilizer subgroup of $q_{\infty}$ in $\mathrm{SU}(2,1$; $\left.\mathbb{Z}\left[\omega_{3}\right]\right)$. Then $\Gamma_{\infty}\left(2,1 ; \mathbb{Z}\left[\omega_{3}\right]\right)$ is generated by the Heisenberg translations $N_{\left(\omega_{3}, \sqrt{3}\right.}$, $N_{(1, \sqrt{3})}$ and the rotation $M_{-\omega_{3}}$.

Proof. We know that any $P \in \Gamma_{\infty}\left(2,1 ; \mathbb{Z}\left[\omega_{3}\right]\right)$ is upper triangular. By Lemma 3.2 there is no dilation component in the Langlands decomposition of $P$, that is,

$$
P=N_{(z, t)} M_{\beta}=\left(\begin{array}{ccc}
1 & -\bar{z} & \frac{1}{2}\left(-|z|^{2}+i t\right) \\
0 & 1 & z \\
0 & 0 & 1
\end{array}\right)\left(\begin{array}{ccc}
1 & 0 & 0 \\
0 & \beta & 0 \\
0 & 0 & 1
\end{array}\right) .
$$

Since $\beta^{6}=1$ the rotation component of $P$ is one of $M_{-\omega_{3}}, M_{\omega_{3}^{2}}=M_{-\omega_{3}}^{2}, M_{-1}=M_{-\omega_{3}}^{3}$, $M_{\omega_{3}}=M_{-\omega_{3}}^{4}, M_{-\omega_{3}^{2}}=M_{-\omega_{3}}^{5}$ or $I=M_{-\omega_{3}}^{6}$. Therefore the rotation component of $P$ in the Langlands decomposition is generated by $M_{-\omega_{3}}$. 
We now consider the Heisenberg translation component of $P$, namely $N_{(z, t)}$. Let $z=a+b \omega_{3}$, where $a, b \in \mathbb{Z}$ since $z \in \mathbb{Z}\left[\omega_{3}\right]$. Then $N_{(z, t)}$ splits as

$$
N_{(z, t)}=N_{\left(a+b \omega_{3}, t\right)}=N_{\left(b \omega_{3}, \sqrt{3} b\right)} \circ N_{(a, \sqrt{3} a)} \circ N_{(0, t-\sqrt{3} a b-\sqrt{3} a-\sqrt{3} b)} .
$$

Here $N_{\left(b \omega_{3}, \sqrt{3} b\right)}$ can be written in the form $N_{\left(b \omega_{3}, \sqrt{3} b\right)}=N_{\left(\omega_{3}, \sqrt{3}\right)}^{b}$ since $b \in \mathbb{Z}$. The Heisenberg translation $N_{(a, \sqrt{3} a)}$ can be written in the form $N_{(a, \sqrt{3} a)}=N_{(1, \sqrt{3})}^{a}$ since $a \in \mathbb{Z}$.

To obtain the equality

$$
N_{(0, t-\sqrt{3} a b-\sqrt{3} a-\sqrt{3} b)}=N_{(0,2 \sqrt{3})}^{(t-\sqrt{3}(a b+a+b)) / 2 \sqrt{3}}
$$

it suffices to show that the number $(t-\sqrt{3}(a b+a+b)) / 2 \sqrt{3}$ is an integer. By Lemma 3.2 the integers $t / \sqrt{3}$ and $|z|^{2}=\left|a+b \omega_{3}\right|^{2}=a^{2}-a b+b^{2}$ have the same parity. It is easy to see that

$$
a^{2}-a b+b^{2}+(a b+a+b)=a(a+1)+b(b+1) \in 2 \mathbb{Z} .
$$

Hence $t / \sqrt{3}$ and $a b+a+b$ have the same parity. It follows that $(t-\sqrt{3}(a b+$ $a+b)) / 2 \sqrt{3}$ is an integer.

The Heisenberg translation $N_{(0,2 \sqrt{3})}$ can be generated by $N_{(1, \sqrt{3})}$ and $M_{-1}$, that is,

$$
N_{(0,2 \sqrt{3})}=\left(N_{(1, \sqrt{3})} \circ M_{-1}\right)^{2} .
$$

Our proposition has now been established.

Proof of Theorem 3.1. Let $G=\left(g_{j k}\right)_{j, k=1}^{3}$ be an element of the group $\operatorname{SU}\left(2,1 ; \mathbb{Z}\left[\omega_{3}\right]\right)$. Since the result is obviously true when $G \in \Gamma_{\infty}$, which is the stabilizer subgroup of $q_{\infty}$, we may assume that $G$ does not belong to the subgroup $\Gamma_{\infty}$.

In this case $g_{31} \neq 0$ by Lemma 3.2 and $G$ maps $q_{\infty}$ to $\left(g_{11} / g_{31}, g_{21} / g_{31}\right)$. Since $G\left(q_{\infty}\right)$ is an element of $\partial \mathbf{H}_{\mathbb{C}}^{2}$, we see that

$$
2 \operatorname{Re}\left(\frac{g_{11}}{g_{31}}\right)=-\left|\frac{g_{21}}{g_{31}}\right|^{2} .
$$

Consider the Heisenberg translation $N_{G\left(q_{\infty}\right)}$ that maps $q_{0}$ to $G\left(q_{\infty}\right)$. Note that the translation $N_{G\left(q_{\infty}\right)}$ does not necessarily lie in the Picard modular group $\mathrm{SU}\left(2,1 ; \mathbb{Z}\left[\omega_{3}\right]\right)$ except when $\left|g_{31}\right|=1$. However, we know that

$$
R N_{G\left(q_{\infty}\right)}^{-1} G=P .
$$

It is well known that the ring $O_{3}=\mathbb{Z}\left[\omega_{3}\right]$ is Euclidean. Thus we may successively approximate $N_{G\left(q_{\infty}\right)}^{-1}$ by Heisenberg translations in the Picard modular group and so decrease the value of $\left|g_{31}\right|^{2} \in \mathbb{Z}$ until it becomes 0 . Therefore $G$ belongs to the subgroup $\Gamma_{\infty}$ by Lemma 3.2 and can be expressed as a product of generators by Proposition 3.3. 
We calculate the entry in the lower left corner of the product

$$
G_{1} \equiv R N_{(z, t)} G=\left(\begin{array}{ccc}
0 & 0 & 1 \\
0 & -1 & -z \\
1 & -\bar{z} & \left(-|z|^{2}+i t\right) / 2
\end{array}\right) G
$$

Now the entry $g_{31}^{(1)}$ lying in the lower left corner of $G_{1}=\left(g_{j k}^{(1)}\right)_{j, k=1}^{3}$ is equal to

$$
\begin{aligned}
g_{31}^{(1)} & =g_{11}-g_{21} \bar{z}+\frac{1}{2}\left(-|z|^{2}+i t\right) g_{31} \\
& =g_{31}\left(\frac{g_{11}}{g_{31}}-\frac{g_{21}}{g_{31}} \bar{z}+\frac{1}{2}\left(-|z|^{2}+i t\right)\right) \\
& =g_{31}\left[\left(\operatorname{Re}\left(\frac{g_{11}}{g_{31}}\right)-\operatorname{Re}\left(\frac{g_{21}}{g_{31}} \bar{z}\right)-\frac{1}{2}|z|^{2}\right)+i\left(\operatorname{Im}\left(\frac{g_{11}}{g_{31}}\right)-\operatorname{Im}\left(\frac{g_{21}}{g_{31}} \bar{z}\right)+\frac{1}{2} t\right)\right] \\
& =g_{31}\left(I_{1}+i I_{2}\right) .
\end{aligned}
$$

We can use (3.1) to simplify $I_{1}$ to

$$
I_{1}=-\frac{1}{2}\left|\frac{g_{21}}{g_{31}}+z\right|^{2}
$$

Let $\left(g_{21} / g_{31}\right)=x+i y$. Since

$$
z=a+b \omega_{3}=\left(a-\frac{1}{2} b\right)+\frac{1}{2} b \sqrt{3} i
$$

we can select two appropriate integers $a$ and $b$ satisfying the conditions $\left|x+\left(a-\frac{1}{2} b\right)\right| \leq$ $\frac{1}{2}$ and $\left|y+\frac{1}{2} b \sqrt{3} i\right| \leq \frac{\sqrt{3}}{4}$. Hence we obtain the upper bound

$$
\left|I_{1}\right| \leq \frac{1}{2}\left(\left(\frac{1}{2}\right)^{2}+\left(\frac{\sqrt{3}}{4}\right)^{2}\right)=\frac{7}{32} .
$$

Choosing some $t$ in $I_{2}$, we calculate the inequality

$$
\left|I_{2}\right|=\left|\operatorname{Im}\left(\frac{g_{11}}{g_{31}}\right)-\operatorname{Im}\left(\frac{g_{21}}{g_{31}} \bar{z}\right)+\frac{1}{2} t\right| \leq \frac{\sqrt{3}}{4}
$$

since $t \in \sqrt{3} \mathbb{Z}$. Therefore we have the following estimate for $g_{31}^{(1)}$ :

$$
\left|g_{31}^{(1)}\right|^{2}=\left|g_{31}\right|^{2}\left|I_{1}+i I_{2}\right|^{2}=\left|g_{31}\right|^{2}\left(I_{1}^{2}+I_{2}^{2}\right) \leq\left|g_{31}\right|^{2}\left[\left(\frac{7}{32}\right)^{2}+\left(\frac{\sqrt{3}}{4}\right)^{2}\right]<\frac{1}{4}\left|g_{31}\right|^{2} .
$$

The preceding inequality tells us that we can reduce the matrix of the transformation $G$ to the matrix of a transformation $G_{n}$ with $g_{31}^{(n)}=0$ by repeating this approximation procedure finitely many times. Moreover, by Lemma 3.2, this condition implies that the transformation $G_{n}$ belongs to the subgroup $\Gamma_{\infty}\left(2,1 ; \mathbb{Z}\left[\omega_{3}\right]\right)$. As we showed in Proposition 3.3, the subgroup $\Gamma_{\infty}\left(2,1 ; \mathbb{Z}\left[\omega_{3}\right]\right)$ can be generated by the Heisenberg translations $N_{\left(\omega_{3}, \sqrt{3}\right)}, N_{(1, \sqrt{3})}$ and the Heisenberg rotation $M_{-\omega_{3}}$. Since the approximation procedure just contains the transformations in $\Gamma_{\infty}\left(2,1 ; \mathbb{Z}\left[\omega_{3}\right]\right)$ and the transformation $R$ the proof of Theorem 3.1 is now complete. 
Remark 3.4. In [4] Falbel and Parker gave the following presentation for the Eisenstein-Picard modular group $\mathrm{PU}\left(2,1 ; \mathbb{Z}\left[\omega_{3}\right]\right)$ :

$$
\left\langle P, Q, R: R^{2}=\left(Q P^{-1}\right)^{6}=P Q^{-1} R Q P^{-1} R=P^{3} Q^{-2}=(R P)^{3}=1\right\rangle .
$$

Moreover, the stabilizer subgroup of infinity $q_{\infty}$ has the presentation $\Gamma_{\infty}=\langle P, Q\rangle$. Here

$$
P=\left(\begin{array}{ccc}
1 & 1 & \omega_{3} \\
0 & \omega_{3} & -\omega_{3} \\
0 & 0 & 1
\end{array}\right), \quad Q=\left(\begin{array}{rrc}
1 & 1 & \omega_{3} \\
0 & -1 & 1 \\
0 & 0 & 1
\end{array}\right), \quad R=\left(\begin{array}{rrr}
0 & 0 & 1 \\
0 & -1 & 0 \\
1 & 0 & 0
\end{array}\right)
$$

By Proposition 3.3 it is clear that $P Q^{-1}=M_{-\omega_{3}}, Q=N_{(1, \sqrt{3})} \circ M_{-\omega_{3}}^{3}$ and

$$
P=M_{-\omega_{3}} \circ Q=M_{-\omega_{3}} \circ N_{(1, \sqrt{3})} \circ M_{-\omega_{3}}^{3} .
$$

This means that the subgroup $\Gamma_{\infty}$ of $\mathrm{PU}\left(2,1 ; \mathbb{Z}\left[\omega_{3}\right]\right)$ can be generated by a Heisenberg translation $N_{(1, \sqrt{3})}$ and a rotation $M_{-\omega_{3}}$. Hence the Picard modular group $\mathrm{PU}\left(2,1 ; \mathbb{Z}\left[\omega_{3}\right]\right)$ is generated by $N_{(1, \sqrt{3})}, M_{-\omega_{3}}$ and $R$.

\section{Acknowledgements}

We would like to thank Professor John Parker, Miaokun Wang and Tiehong Zhao for pointing out errors in the first manuscript as well as many helpful suggestions. We would like to thank the referee for his or her valuable suggestions.

\section{References}

[1] L. Bao, C. Colonello, A. Kleinschmidt, B. Nillson and D. Persson, 'Instanton correction to the universal hypermultiplet and automorphic forms on SU(2, 1)', Commun. Numer. Theory Phys. 4 (2010), 187-266.

[2] E. Falbel, G. Francsics, P. D. Lax and J. R. Parker, 'Generators of a Picard modular group in two complex dimensions', Proc. Amer. Math. Soc. 139 (2011), 2439-2447.

[3] E. Falbel, G. Francsics and J. R. Parker, 'The geometry of the Gauss-Picard modular group', Math. Ann. 349 (2011), 459-508.

[4] E. Falbel and J. R. Parker, 'The geometry of the Eisenstein-Picard modular group', Duke Math. J. 131 (2006), 249-289.

[5] G. Francsics and P. Lax, 'A semi-explicit fundamental domain for a Picard modular group in complex hyperbolic space’, Contemp. Math. 238 (2005), 211-226.

[6] G. Francsics and P. Lax, 'An explicit fundamental domain for a Picard modular group in complex hyperbolic space', Preprint, 2005, pp. 1-25, arXiv:math/0509708.

[7] W. M. Goldman, Complex Hyperbolic Geometry (Oxford University Press, Oxford, 1999).

[8] G. H. Hardy and E. M. Wright, An Introduction to the Theory of Numbers (Oxford University Press, Oxford, 1954).

[9] R.-P. Holzapfel, 'Invaiants of arithmetric ball quotient sufraces', Math. Nachr. 103 (1981), 117-153.

[10] J. R. Parker, Notes on Complex Hyperbolic Geometry (Preliminary version, 2003).

[11] T. Zhao, 'Generators for the Euclidean Picard modular groups', Trans. Amer. Math. Soc., to appear. 
JIEYAN WANG, College of Mathematics and Economics, Hunan University, Changsha 410082, PR China e-mail: jywang@hnu.edu.cn

YINGQING XIAO, College of Mathematics and Economics, Hunan University, Changsha 410082, PR China e-mail: ouxyq@yahoo.cn

BAOHUA XIE, College of Mathematics and Economics, Hunan University, Changsha 410082, PR China e-mail: xiebh@gmail.com 